\title{
Mise à jour de l'inventaire des Chironomidés (Diptera) connus du Maroc
}

\author{
K. Kettani ${ }^{1}$ \\ T. El Ouazzani ${ }^{1}$ \\ D. Calle Martinez ${ }^{2}$
}

Mots-clés : Diptera, Chironomidae, faunistique, Maroc.

Un dernier inventaire récapitulant la totalité des espèces ou taxa de Chironomidés signalés jusqu'en 2001 est présenté avec la répartition géographique de chaque espèce au sein du territoire marocain. Le total des espèces ou taxa actuellement connues du Maroc est de 317.

La répartition de cette faune entre les différentes aires géographiques définies du Maroc est distincte. 163 espèces sont recensées dans le Rif et 256 dans l'Atlas (Moyen-Atlas + Haut-Atlas + Anti-Atlas).

\section{Update on the Chironomid (Diptera) inventory recorded for Morocco}

Keywords : Diptera, Chironomidae, fauna, Marocco.

A complete inventory of data on Chironomidae (Diptera) reported up to the year 2001 is presented together with the distribution of each of species within the Moroccan territory. The total number of species or taxa now recorded for Morocco is 317 .

The distribution of this fauna over the different defined areas of Morocco is distinct. The Rif contains 163 species while the Atlas contains 256 species.

\section{Introduction}

Le présent travail présente un bilan complet, sauf erreurs ou omissions, de la faune chironomidienne recensée actuellement au Maroc.

Le premier inventaire faunistique des Chironomidés du Maroc paru en 1987 avait recensé 134 espèces (Azzouzi \& Laville 1987), le second en 1992 (Azzouzi et al. 1992) a porté ce chiffre à 223 espèces. Ces đonnées intéressent essentiellement le centre et le sud du Maroc. Avec de nouvelles récoltes effectuées dans le nord et plus particulièrement dans le région du Rif (Kettani et al. 1994, 1995, 1997) et à partir de certaines données non publiées dans les inventaires précédents, nous pré-

1. Département de Biologie, Faculté des Sciences, Université Abdelmalek Essaadi, B.P. 2121, Tétouan, Maroc.

E. mail : kettanikawtar@hotmail.com

2. IES «Los Manantiales». Departamento de Biologia y Geologia.

29620. Torremolinos (Màlaga), España.

E.mail : demetriocalle@hotmail.com sentons un inventaire des Chironomidés recensés sur tout le territoire marocain.

En outre, dans le but de ressortir l'intérêt biogéographique des espèces recensées au sein dụ territoire marocain, nous signalons leur répartition selon quatre régions : le Rif, le Moyen-Atlas, le Haut-Atlas et l'AntiAtlas.

\section{Aires géographiques}

Le territoire marocain a été subdivisé en quatre aires géographiques principales : le Rif, le Moyen-Atlas, le Haut-Atlas et 1'Anti-Atlas (Fig. 1). Les études faunistiques consacrées aux Chironomidés du Maroc concernent l'une ou l'autre de ces aires. Nous présentons de façon très succincte ces différentes aires avec leurs sites de prospections numérotés et les références bibliographiques concernant ces différents sites d'étude.

\subsection{Le Rif}

Le Rif correspond à la pointe la plus septentrionale du Maroc entre la Méditerranée au N-E et l'océan Atlantique au N-O. Sur le plan géologique, c'est la seule 


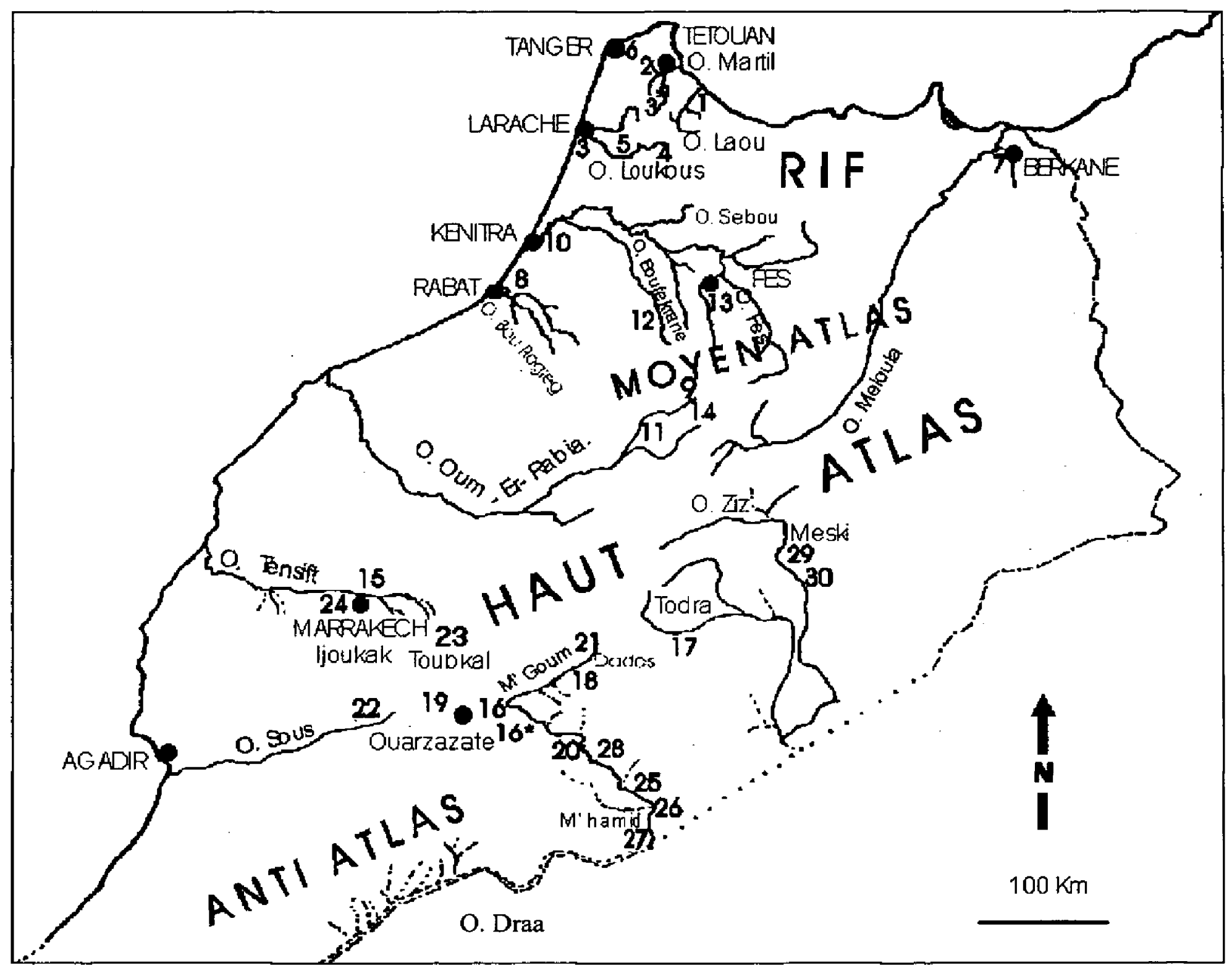

Fig. 1 : Découpage du Maroc en quatre principales zones de récolte : Rif, Moyen-Atlas, Haut-Atlas et Anti-Atlas.

Fig. 1 : The four main zones where collections have been made in Morocco : Rif, Moyen-Atlas, Haut-Atlas and Anti-Atlas.

chaîne marocaine issue de I'orogénie alpine et plusieurs de ses faciès ont plus d'affinités avec ceux de l'Andalousie qu'avec ceux du reste du Maroc (Thauvin 1971). Ses caractéristiques géologiques, géographiques, pluviométriques lui confèrent une grande variabilité dans la potentialité des ressources en eaux souterraines et une dissymétrie dans l'hydrographie entre le versant méditerranéen (oueds courts à pente forte) et le versant atlantique (oueds longs, avec nombreux affluents).

Les cours d'eau prospectés sur la rive méditerranéenne sont les oueds Laou et Martil et sur le versant atlantique, quelques prospections très fragmentaires concernent essentiellement des descriptions d'espèces nouvelles sur les oueds Loukous et Marhar.
1 : Oued Laou (35-1300 m) : Kettani et al. 1994, 1997

2 : Oued Martil (25-425 m) : Kettani et al. 1995

3: Environs de Larache : Azzouzi \& Laville 1987

*3 : Environs de Tétouan : Azzouzi \& Laville 1987

4 : Estuaire de l'Oued Loukous : Azzouzi \& Laville 1987

5 : Retenue El Makhazine (357 m) : Azzouzi \& Laville 1987

6: Mare Kenna (Estuaire de l'Oued Marhar), 25 Km SO Tanger : Azzouzi \& Laville 1987

7 : Environs de Berkane : Azzouzi \& Laville 1987

\subsection{Les Atlas}

Les Atlas s'opposent au domaine rifain par leur rattachement à l'Afrique occidentale avec surtout une in- 
fluence atlantique. C'est le pays de la haute montagne, relativement bien arrosé par les pluies d'automne et du printemps et les orages de l'été, et recouvert de neige en hiver. La végétation y est beaucoup plus abondante et diversifiée que dans les régions plus basses du Maroc. La forêt y est à peu près préservée. Ces régions sont les châteaux d'eau du Maroc. De là proviennent les grands oueds atlantiques - Sebou, Bou-Regreg, Oum-erRbia, Tensift, Sous et Draa -, l'oued Moulouya qui se jette en Méditerranée et ceux comme le Ziz ou le Rheris qui se perdent dans les bassins présahariens.

\section{- Le Moyen-Atlas}

Le Moyen-Atlas, le plus septentrional, se sépare du Haut-Atlas dans la région de Beni-Mellal et s'étend en direction du nord-est jusqu'à Taza.

Les cours d'eau prospectés dans cette aire sont l'oued Sebou et ses affluents, l'oued Bouferkane et l'oued Fès ; certains lacs de haute altitude où ont été trouvés des holotypes d'espèces nouvelles. Toutefois, ces prospections dans le Moyen-Atlas se réservent d'être intensives. Les différents sites de récoltes sont les suivants :

8 : Merja Sidi Bou Ghaba : Rhamdani \& Tourenq 1982

9 :Lac Aguelman Axighx (1510 m) : Azzouzi \& Laville 1987

10 : Oued Sebou (180-221m) : Azzouzi \& Laville 1987 et Naya 1988

11 : Oued Oum-er-Rbia (1280-1500 m) : Azzouzi \& Laville 1987

12 : Oued Bouferkane (340-750 m) : Azzouzi \& Laville 1987

13 : Oued Fès (400 m) : Azzouzi \& Laville 1987

14 : Lac Ouiouane $(1635 \mathrm{~m})$ : Azzouzi \& Laville 1987

\section{- Le Haut-Atlas}

Le Haut-Atlas est le massif montagneux le plus important du Maroc qu'il traverse de part en part en direction WSW-ENE. Il sépare le Maroc en plateaux et plaines atlantiques au nord et à l'ouest et le domaine saharien au sud et au sud-est.

Dans cette aire, à l'exception de l'oued Tensift et des Khettaras de Marrakech qui ont été étudiés intensivement, les autres sites furent prospectés de manière assez fragmentaire. Ces autres sites montrent une grande variété de milieux de haute altitude, des lacs, des sources, des gorges de montagne, des eaux souterraines,... Ces différents milieux sont récapitulés ci-dessous :

15 : Oued Tensift (300-1600 m) : Azzouzi et al. 1992
16: Ouarzazate (près du lac de retenue) à $1140 \mathrm{~m}$ : Azzouzi et al. 1992

* 16: Ouarzazate (capture à la lumière) à $1160 \mathrm{~m}$ : Azzouzi et al. 1992

17 : Gorges de Todra (Sud Haut-Atlas) à $1400 \mathrm{~m}$ : Azzouzi \& Laville 1987

18 : Gorges de Dadès, près Imdiazen (Sud Haut-Atlas) à $1900 \mathrm{~m}$ : Azzouzi \& Laville 1987

19 : Vallée Asit Amini, près Amergane (Sud HautAtlas) à $1540 \mathrm{~m}$ : Azzouzi et al. 1992

20 : Ait Saoun, entre Ouarzazate et Zagora : Azzouzi et al. 1992

21 : Source de M'Goum (Sud Haut-Atlas) à $2500 \mathrm{~m}$ : Azzouzi \& Laville 1987

22 : Lac Tamhda (2800 m) : Azzouzi \& Laville 1987

23 : Massif de Toubkal : Oukaimeden $(2500 \mathrm{~m})$ : Azzouzi \& Laville 1987

24 : Khettaras de Marrakech (410-700 m) : El Mezdi \& Giudicelli 1985

\section{- L'Anti-Atlas}

L'Anti-Atlas représente le sud du Maroc, c'est le domaine présaharien s'étendant au nord de l'oued Draa, formant un large bombement parallèle au Haut-Atlas. Il n'est séparé de ce dernier que par un ensemble de dépressions formant le sillon sud-atlasique. Ce sillon est occupé par de nombreux oasis groupés autour des oueds descendants du Haut-Atlas : le Sous à l'ouest, le Haut Draa et le Dadès dans la dépression de Ouarzazate. Les prospections dans cette aire demeurent très rares :

25 : Tissint, Moyen Draa : Azzouzi et al. 1992

26 : Dra-Tal (2500-3000 m) : Azzouzi \& Laville 1987

27 : N'Hamid Dra-Tal : Azzouzi \& Laville 1987

28: Vallé de Draa : Azzouzi et al. 1992

28 : Oasis Meski (1160 m) : Azzouzi et al. 1992

28 : Oasis Meski : Azzouzi et al. 1992

\section{Résultats et discussion}

Le tableau 1 récapitule l'inventaire actuel de la faune chironomidienne du Maroc, répartie selon les 30 sites de récolte de chaque espèce dans les différentes aires délimitées du Maroc.

Ce nouvel inventaire porte à 317 le total des espèces ou taxa actuellement recensés du Maroc.

Le tableau 2 présente la répartition ainsi que l'évolution du nombre d'espèces recensées dans les différentes sous-familles ou tribus de Chironomidés en 1987, en 1992 et en 2001. 
Tableau 2. Evolution dans le temps du nombre d'espèces ou taxa de Chironomidés recensés au Maroc entre 1987 et 2001.

Table 2. Increase in of the number of species or taxa of Chironomids reported in Morocco between 1987 and 2001.

\begin{tabular}{lccc}
\hline & $\mathbf{1 9 8 7}$ & $\mathbf{1 9 9 2}$ & $\mathbf{2 0 0 1}$ \\
\hline Prodiamesinae & - & 1 & 1 \\
Diamesinae & 8 & 12 & 21 \\
Podonominae & - & 1 & 1 \\
Tanypodinae & 15 & 31 & 45 \\
Orthocladiinae & 42 & 80 & 107 \\
Chironomini & 49 & 62 & 96 \\
Tanytarsini & 23 & 36 & 46 \\
Total & 137 & 223 & 317 \\
\hline
\end{tabular}

Cette nouvelle mise à jour a permis d'ajouter 94 espèces de Chironomidés à la liste de 1992. En outre, ce nombre révèle aussi l'existence d'une importante potentialité de la diversité spécifique des Chironomidés au nord du Maroc. Cette aire, comme le reste du Maroc, mérite d'être étudiée plus intensivement.

L'analyse de la répartition des données faunistiques des Chironomidés montre qu'elles se répartissent dans l'ensemble des régions géographiques du Maroc, mais de façon non uniforme. Cela revient tout d'abord aux différences de milieux prospectés d'une région à l'autre et surtout à la fréquence variable dont ces milieux ont été prospectés. Ainsi parmi les 317 espèces ou taxa recensés, le Rif renferme 163 espèces $(51 \%)$ dont 60 espèces sont exclusives de cette région. L'Atlas renferme 256 espèces réparties entre 136 espèces (43\%) rencontrées au Moyen-Atlas avec 65 espèces exclusives ; 128 espèces $(40 \%)$ recensées au Haut-Atlas avec 70 espèces exclusives et seulement 22 espèces (7\%) dans l'Anti-Atlas mais avec 11 espèces exclusives de ce domaine présaharien.

En Outre, au sein de chaque aire, d'importantes différences du nombre d'espèces récoltées s'observent d'une localité à une autre. Dans le Rif, seuls l'oued Laou et l'oued Martil atteignent respectivement 113 et 101 espèces et s'avèrent les localités les mieux inventoriées. Dans le Moyen-Atlas, seuls 1'oued Sebou, l'oued Oum-er-Rbia, l'oued Bouferkane, l'oued Fès et la Merja de Sidi Bou Ghaba atteignent respectivement $80,38,31,28$ et 18 espèces. Le maximum d'espèces dans le Haut-Atlas est atteint dans l'oued Tensift (89 espèces) et dans les Khettaras de Marrakech
(24 espèces). Quant à l'Anti-Atlas, le maximum est atteint dans le Dra-Tal avec seulement 11 espèces.

Les lacs prospectés au Maroc sont le lac Aguelman Axighx, le lac Ouiouane du Moyen-Atlas et le lac Tamhda du HautAtlas avec respectivement 3, 1 et 2 espèces recenisées.

Quant aux sources prospectées, très peu d'espèces ont été récoltées. La source de Maggou à $1300 \mathrm{~m}$ (oued Laou) renferme 22 espèces, celle de $M^{\prime}$ 'Goum à $2500 \mathrm{~m}$ (oued Draa) ne renferme qu'une seule espèce (Diamesa hamaticornis).

Au Sud de l'Anti-Atlas qui représente le domaine présaharien, une seule espèce a été recoltée : Paramerina mauretanica.

Entre ces quatre massifs montagneux, une seule espèce est commune : Cardiocladius fuscus, signalée du Potamon avec une large répartition en Europe (Laville 1981). Cette exceptionnelle rareté en espèces communes entre les différentes régions du Maroc témoigne une fois de plus de l'ample diversité en caractéristiques hydrologiques, morphométriques, géologiques, géographiques, climatologiques et floristiques. Ceci explique la grande diversité dans le peuplement chironomidien des différentes régions du territoire marocain et ses variations d'une région à l'autre.

L'analyse de la composition faunistique des Chironomidés dans ces différentes aires (Fig. 2) montre que le Rif renferme le plus grand nombre d'espèces en Orthocladiinae, Tanypodinae, Chironomini et Tanytarsini ; le Moyen-Atlas renferme un maximum d'espèces en Diamesinae, Prodiamesinae et Chironomini tandis

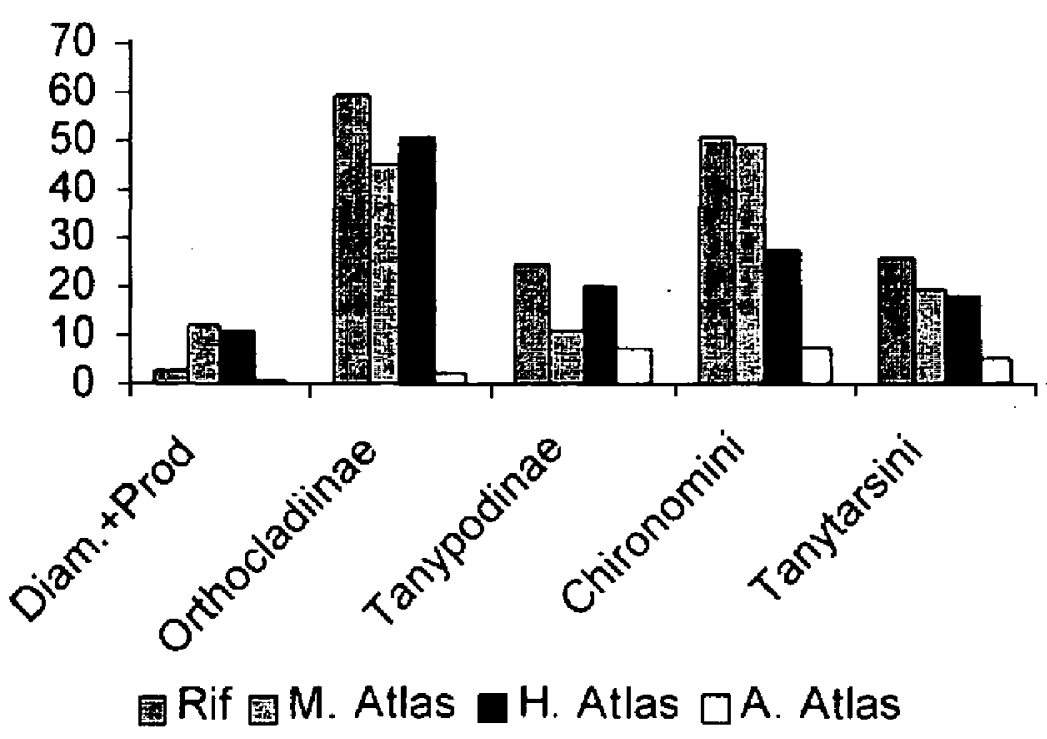

Fig. 2. Total des espèces de Chironomidés des principales sous-familles et tribus recensées dans les 4 aires géographiques du Maroc.

Fig. 2 : Total number of species of the main taxa of Chironomids reported in the 4 areas of Morocco. 
Tableau 1. Inventaire des Chironomidés recensés jusqu'en 2001 au Maroc.

Table 1. Update of the inventory of Chironomids reported up to 2001 in Morocco.

\begin{tabular}{|c|c|c|c|c|}
\hline & RIF & M. ATLAS & H. ATLAS & A. ATLAS \\
\hline \multicolumn{5}{|l|}{ PRODIAMESINAE } \\
\hline Prodiamesa olivacea (Mg.) & 1 & 10 & 15 & \\
\hline \multicolumn{5}{|l|}{ DIAMESINAE } \\
\hline Boreoheptagyia legeri (Goetghebuer) & & & 15 & \\
\hline Boreoheptagyia punctulata (Goetghebuer) & & & $18(2550 \mathrm{~m})$ & \\
\hline Diamesa aberrata Lundbeck & & & & $26(3350 \mathrm{~m})$ \\
\hline Diamesa bertrami Edwards & & & 17 & \\
\hline Diamesa hamaticomis Kieffer & & & 21 & \\
\hline Diamesa insignipes Kieffer & & 10 & 17 & \\
\hline Diamesa? latitarsis (Goetghebuer) & & & Haut-Atlas ? & \\
\hline Diamesa thienemanni Halvorsen & & 10 & & \\
\hline Diamesa vaillanti Serra-Tosio & & & 15 & \\
\hline Diamesa veletensis Serra-Tosio & & & 17 & \\
\hline Diamesa? zemyi Edwards & & & 15 & \\
\hline Diamesa sp1 & & 10 & & \\
\hline Diamesa sp2 & & 10 & & \\
\hline Odontormesa fulva (Kieffer) & & 11 & & \\
\hline Potthastia gaedii (Mg.) & 1,2 & $11,12,13$ & & \\
\hline Protanypus morio (Zett.) & & 10 & & \\
\hline Pseudodiamesa branickii (Nowicki) & & 10 & & \\
\hline Pseudodiamesa nivosa (Goetghebuer) & & 10 & & \\
\hline Sympotthastia zavreli Pagast & 1,2 & & 15 & \\
\hline Syndiamesa hygropterica Kieffer & & 10 & & \\
\hline Syndiamesa sp & & 10 & & \\
\hline \multicolumn{5}{|l|}{ TANYPODINAE } \\
\hline Ablabesmyja ebbae L. & $3^{*}$ & & & \\
\hline Ablabesmyia longistyla Fitlkau & 1 & & 15,24 & \\
\hline Ablabesmyia monilis (L.) & & 10,13 & & \\
\hline Ablabesmyia sp & & 10 & & \\
\hline Clinotanypus sp & & 10 & & \\
\hline Conchapelopia melanops (Mg.) & 1 & & & \\
\hline Conchapelopia viator (Kieffer) & 1,2 & & 18 & \\
\hline Conchapelopia sp & & & 24 & \\
\hline Larsia atrocincta (Goetghebuer) & 1 & & & \\
\hline Larsia? curticalcar (Kieffer) & & & 15 & \\
\hline Macropelopia nebulosa (Mg.) & 1 & & 15 & \\
\hline Nilotanypus dubius (Mg.) & 1,2 & & 15 & \\
\hline Paramenina cingulata (Walker) & 1,2 & & 15 & \\
\hline Paramerina divisa (Walker) & 1 & & & \\
\hline Paramerina spec. Griechenland Fittkau & 1,2 & & & \\
\hline Paramerina mauretanica Fittkau & & & & Sahara Atlas \\
\hline Paramerina Pe 1 Langton 84 & & 11 & & \\
\hline Pentaneurella sp Ourika & & & 15 & \\
\hline Procladius anomalus Kieffer & & 10 & & \\
\hline Procladius brevipetiolatus (Goetgebuer) & & & 24 & 29 \\
\hline Procladius choreus (Mg.) & 1,2 & 8 & 24 & \\
\hline Procladius noctivagus (Kieffer) & & & $16^{*}$ & \\
\hline Procladius sagittalis (Kieffer) & 1 & & & \\
\hline Procladius Pe 3 Langton 91 & 1,2 & & & \\
\hline Psectrotanypus varius (Fabricius) & 1 & & & \\
\hline Rheopelopia maculipennis (Zett.) & 1,2 & 10,11 & 15 & \\
\hline
\end{tabular}


Tableau 1. Suite.

Table 1. Continued.

Rheopelopia murrayi Dowling

Rheopelopia omata (Mg.)

Tanypus? brevipalpis Kieffer

Tanypus kraatzi (Kieffer)

Tanypus punctipennis $\mathrm{Mg}$.

Telmatopelopia nemorum (Goetghebuer)

Telopelopia fascigera (Vemeaux)

Telopelopia maroccana Murray

Thienemannimyia berkanea Dowling

Thienemannimyia choumara Dowling

Thienemannimyia? laeta (Mg.)

Thienemannimyja lentiginosa (Fries)

Thienemannimyia nonthumbrica (Edwards)

Trissopelopia Jongimana (Staeger)

Xenopelopia nigricans (Goetghebuer)

Zavrelimyia ?barbatipes (Kieffer)

Zavrelimyia berberi Fittkau

Zavrelimyia melanura (Mg.)

Zavrelimyia sp

1,2
7
1
1
3
7
7
1,2
1

13

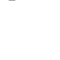

30

15,24

11

10

PODONOMINAE

Paraboreochlus minutissimus (Strobl)

\section{ORTHOCLADINAE}

Brillia bifida (Kieffer)

Brillia flavifrons Johannsen

Bryophaenocladius subvemalis (Edwards)

1

Cardiocladius capucinus (Zett.)

Cardiocladius fuscus (Kieffer)

Chaetocladius gr. acuticomis

Chaetocladius gr. vitellinus

Corynoneura camiana Edwards

Corynoneura lacustris Edwards

Corynoneura lobata Edwards

Corynoneura Pe 2 Langton 91

Corynoneura sp

Cricotopus (C) albiforceps (Kieffer)

Cricotopus (C) annulator Goetghebuer

Cricotopus (C) beckeri Hirvenoja

Cricotopus (C) bicinctus ( $\mathrm{Mg}$.)

C. (C) levantinus Moubayed \& Hirvenoja

Cricotopus (C) pallidipes Edwards

Cricotopus (C) similis Goetghebuer

Cricotopus (C) triannulatus (Macquart)

Cricofopus (C) trifascia Edwards

Cricotopus (C) vierriensis Goetghebuer

Cricotopus (I) brevipalpis Kieffer

Cricotopus (l) laetus Hirvenoja

Cricotopus (I) omatus (Mg.)

Cricotopus (I) sytvestris (Fabricius)

Eukiefferiella bedmari Vilchez \& Laville

Eukiefferiella brehmi Gowin

Eukiefferiella brevicalcar (Kieffer)

Eukiefferiella claripennis (Lundbeck)

Eukiefferiella clypeata (Kieffer)

Eukiefferiella coerulescens (Kieffer)
2

1,2

\section{1,2}

$10,11,12$

12

11

$10,11,12$

10

10

1

1,2

\section{5}


Tableau 1. Suite.

Table 1. Continued.

Eukiefferiella cyanea Thienemann

Eukiefferiella devonica (Edwards)

Eukiefferiella fttkaui Lehmann

Eukiefferiella fuldensis Lehmann

Eukiefferiella gracei (Edwards)

Eukiefferiella ikleyensis (Edwards)

Eukiefferiella lobifera Goetghebuer

Eukiefferiella? minor (Edwards)

Eukiefferiella similis Goetghebuer

Eukiefferiella tirolensis Goetghebuer

Eukiefferiella sp

Halocladius varians (Staeger)

Heleniella omaticollis (Edwards)

Heterotrissocladius marcidus (Walker)

Krenosmittia boreoalpina (Goetghebuer)

Krenosmittia camptophleps (Edwards)

Krenosmittia halvorseni (Cranston\&Saether)

Limnophyes minimus Mg.

Limnophyes ninae Saether

Metriocnemus fuscipes (Mg.)

Metriocnemus hygropetricus Kieffer

Metriocnemus obscuripes (Holmgren)

Orthocladinae gen.? sp? Pe1 Langton 91

(cf. Krenosmittia hispanica Wülker)

Nanocladius balticiss (Palmén)

Nanocladius rectinervis (Kieffer)

Orthocladius (E) ashei Soponis

Orthocladius (E) fuscimanus (Kieffer)

Orthocladius (E) Juteipes Goetghebuer

Orthocladius (E) rivulorum Kieffer

Orthocladius (E) ruffoi Rossaro

Orthocladius (E) thienemanni Kieffer

Orthocladius (E) $\mathrm{sp}$

Orthocladius (O) frigidus (Zett)

Orthocladius (O) oblidens (Walker)

Orthocladius (O) obumbratus Johannsen

Orthocladius (O) rubicundus (Mg.)

Orthocladius (O) $\mathrm{sp}$

Orthocladius sp

Paracricotopus niger (Kieffer)

Parakiefferiella coronata (Edwards)

Parakiefferiella wuelkeri Moubayed

Parametriocnemus stylatus (Kieffer)

Parametriocnemus Pe1 Langton1991

Parametriocnemus sp Rheghaya

Paratrichocladius micans (Kieffer)

Paratrichocladius rufiventris (Mg.)

Paratrichocladius skiwithensis (Edwards)

Paratrissocladius excerptus (Walker)

Parorthocladius nudipennis (Kieffer)

Psectrocladius barbatipes Kieffer

Psectrocladius brehmi Kieffer

Psectrocladius limbatel/us (Holmgren)

Psectrocladius obvius (Walker)

Psectrocladius octomacujatus Wülker

Psectrocladius sordidellus (Zett.)

\begin{tabular}{|c|c|c|}
\hline 2 & & Haut Atlas \\
\hline 1 & 13 & \\
\hline & & 15 \\
\hline & 11 & 15 \\
\hline 1 & & \\
\hline & 11 & 15 \\
\hline & 11,13 & \\
\hline & & Haut Atla: \\
\hline & $10,11,12,13$ & \\
\hline 1 & & 15 \\
\hline & 12 & \\
\hline 1 & 8 & \\
\hline 1,2 & & \\
\hline & 10 & \\
\hline 2 & & \\
\hline & & 15 \\
\hline 2 & & \\
\hline & 8,9 & \\
\hline 2 & & \\
\hline & & 15 \\
\hline & & 15 \\
\hline & & 15 \\
\hline & & 15 \\
\hline 2 & & \\
\hline 1,2 & 11,12 & \\
\hline 1,2 & $10,12,13$ & \\
\hline 1.2 & & 15 \\
\hline & 11,13 & \\
\hline 1,2 & & \\
\hline 1,2 & 11 & \\
\hline 1,2 & & 15 \\
\hline & 10 & \\
\hline 1 & 10 & 22 \\
\hline & & 15 \\
\hline 1,2 & 11,13 & \\
\hline 1,2 & $10,11,12$ & 15 \\
\hline & 10 & \\
\hline & & 24 \\
\hline 1.2 & 12,13 & \\
\hline & & 15 \\
\hline 2 & & 15 \\
\hline 1.2 & $11,12,13$ & \\
\hline 2 & & \\
\hline & & 15 \\
\hline 1.2 & & \\
\hline 2 & $10,11,12,13$ & 15,24 \\
\hline & & 15 \\
\hline 1 & & \\
\hline & & 15 \\
\hline 1,2 & & \\
\hline 2 & & \\
\hline & & 22 \\
\hline & 10 & 15 \\
\hline 2 & & \\
\hline & 14 & \\
\hline
\end{tabular}


Tableau 1. Suite.

Table 1. Continued.

Psectrocladius ventricosus Kieffer

Pseudorthocladius berthelemyi Moubayed

Pseudorthocladius curtistylus (Goetghebuer)

Pseudosmittia recta (Edwards)

Rheocricotopus atripes (Kieffer)

Rheocricotopus chalybeatus (Edwards)

Rheocricotopus effusus (Walker)

Rheocricotopus fuscipes (Kieffer)

Rheocricotopus glabricollis ( $\mathrm{Mg}$.)

Rheocricotopus tirolus Lehmann

Synorthocladius semivirens (Kieffer)

Thienemanniella acuticomis (Kieffer)

Thienemanniella majuscula (Edwards)

Thienemanniella vittata (Edwards)

Thienemanniella $\mathrm{sp}$

Trissocladius brevipalpis Kieffer

Trissocladius sp Tensift

Tvetenia bavarica (Goetghebuer)

Tvetenia calvescens (Edwards)

Tvetenia verralli (Edwards)

$$
\begin{array}{r}
1,2 \\
1,2 \\
\\
1,2 \\
1
\end{array}
$$$$
10
$$$$
10,11,12
$$$$
10
$$$$
12
$$

Chironomus annularius auct.

Chironomus aprilinus $\mathbf{M g}$.

Chironomus bemensis Kiötzli

Chironomus calipterus Kieffer

Chironomus luridus Strenzke

Chironomus piger Strenzke

Chironomus plumosus (L.)

Chironomus riparius $\mathrm{Mg}$.

Chironomus salinarius Kieffer

Chironomus tentans Fabricius

Cryptochironomus albofasciatus (Staeger)

Cryptochironomus psittacinus (Mg.)

Cryptochironomus rostratus Kieffer

Cryptochironomus Pe 5 Langton 91

Cryptochironomus sp

Demicryptochironomus vulneratus (Zett.)

Demicryptochironomus (irmakia) Pe1Langton

Dicrotendipes collarti Goetghebuer

Dicrotendipes cordatus Kieffer

Dicrotendipes fusconotatus (Kieffer)

Dicrotendipes nervosus (Staeger)

Dicrotendipes pallidicomis (Goetghebuer)

Dicrotendipes peningueyanus Kieffer

Dicrotendipes septemmaculatus (Becker)

Endochironomus sp cf albimanus (Mg.)

Endochironomus tendens (Fabricius)

Glyptotendipes gripekoveni (Kieffer)

Glyptotendipes pallens (Mg.)

Glyptotendipes viridis (Macquart)

Glyptotendipes sp A

Glyptotendipes sp B

Hamischia curtilamellatus (Malloch)
8,10

\section{8}

8

8

$1,5 ? \quad 8,10$

24

$8,10,12,13$

8

10

1

2

$10,11,12,13 \quad 15,24$

10

24

15
10

12

8

$1,2,3$
10

10

5

10

10

10

10

$1,210,13$ 
Tableau 1. Suite.

Table 1. Continued.

Hamischia fuscimana Kieffer

Hamischia sp

Harnischia ? n. sp Reiss

Kiefferulus tendipediformis (Goetghebuer)

KJoosia pusilla (L.)

Lauterbomiella agrayloides (Kieffer)

Microchironomus deribae Freeman

Microchironomus lendii (Kieffer)

Microchironomus tener (Kieffer)

Microtendipes britteni (Edwards)

Microtendipes confinis (Mg.)

Microtendipes difinis (Edwards)

Microtendipes pedellus (De Greer)

Microtendipes sp

Parachironomus frequens (Johannsen)

Parachironomus parilis (Walker)

Paracladopelma camptolabis (Kieffer)

Paracladopelma ? galaptera (Townes)

Paracladopelma graminicolor (Kieffer)

Paracladopelma laminata (Kieffer)

Paracladopelma mikiana (Goetghebuer)

Paralauterborniella nigrohalteralis (Malloch)

Paratendipes albimanus (Mg.)

Paratendipes striatus (Kieffer)

Paratendipes sp

Pentapedilum sp

Phaenopsectra flavipes (Mg.)

Polypedilum (Pent) nubens (Edwards)

Polypedilum (Pent) sordens (v.d.W.)

Polypedilum (Pent) uncinatum (Goetghebuer)

Polypedilum (P) albicome (Mg.)

Polypedilum $(P)$ arundineti (Goetghebuer)

Polypedilum (P) convictum (Walker)

Polypedilum $(P)$ cultellatum Goetghebuer

Polypedilum (P) laetum (Mg.)

Polypedilum (P) nubeculosum (Mg.)

Polypedilum (P) nubifer (Skuse)

Polypedilum ( $P$ ) pedestre (Mg.)

Polypedilum $(P)$ ? pharao Kieffer

Polypedilum $(P)$ ruandae Freeman

Polypedilum ( $P$ ) tridens Freeman

Polypedilum (T) aegyptium Kieffer

Polypedilum (T) acifer Townes

Polypedilum ( $T$ ) bicrenatum Kieffer

Polypedilum (T) pullum (Zett.)

Polypedilum (T) quadriguttatum Kieffer

Polypedilum ( $T$ ) scalaenum (Schrank)

Polypedilum (T) tetracrenatum Hirvenoja

Polypedilum Pe 1 Langton 91

Polypedilum ontario group sp

Polypedilum sp

Rheomus alatus Laville \& Reiss

Rheomus yahiae Laville \& Reiss

Saetheria sp

Stictochironomus caffrarius (Kieffer)

Stictochironomus maculipennis (Mg.)

\begin{tabular}{|c|c|c|c|}
\hline $1,2,5$ & 12 & & \\
\hline & 10 & & \\
\hline & & & 29 \\
\hline $3^{*}$ & 8 & & \\
\hline & & 15 & \\
\hline & 10 & & \\
\hline & 8 & & \\
\hline & & & 25 \\
\hline 1 & & 15 & \\
\hline 1,2 & & & \\
\hline 1 & & 15 & \\
\hline & & & 26 \\
\hline $3^{*}$ & 10 & 24 & \\
\hline & 10 & & \\
\hline 2 & & & \\
\hline 2,3 & 9 & & \\
\hline 1,2 & & & \\
\hline & & 16,17 & \\
\hline & & 15 & \\
\hline & & & 26 \\
\hline 2 & & 15 & \\
\hline & $10,12,13$ & & \\
\hline 1,2 & 10 & & \\
\hline & & 24 & \\
\hline & 10 & & \\
\hline & 10 & & \\
\hline 1,2 & & & \\
\hline 1,2 & 10 & & \\
\hline 1 & & & \\
\hline & 12,13 & & \\
\hline 2 & 10 & & \\
\hline 1 & & 15 & \\
\hline $1,2,3$ & 10,12 & & \\
\hline 2 & 11 & & \\
\hline & & 15 & \\
\hline $\begin{array}{c}1,2 \\
3\end{array}$ & 10 & & \\
\hline 1,2 & 12 & & \\
\hline 3 & 8,10 & & \\
\hline & & 24 & \\
\hline & & 24 & \\
\hline $1,2,3$ & & 17,23 & \\
\hline 1 & $10,12,13$ & & 26 \\
\hline & 10 & & \\
\hline & 11,12 & 15,24 & \\
\hline 2 & 10 & & \\
\hline 2 & & & 26 \\
\hline & & 15 & \\
\hline 2 & & 15 & \\
\hline 2 & & & \\
\hline & & 24 & \\
\hline & & 15 & \\
\hline & 13 & & \\
\hline 2 & & & \\
\hline & 13 & & \\
\hline 1,2 & 10 & & \\
\hline
\end{tabular}


Stictochironomus pictulus (Fabricius) Stictochironomus sticticus (Fabricius) Stictochironomus reissi Cranston Stictochironomus $\mathrm{Pe} 2$ Langton 1991 Xenochironomus xenolabis Kieffer Chironomini gen.? sp.? Pe 3 Langton 91 Chironomini gen.? sp.? Pe 4 Langton 91

Tanytarsini

Cladotanytarsus atridorsum Kieffer Cladotanytarsus capensis (Freeman) Cladotanytarsus ecristatus Reiss Cladotanytarsus mancus (Walker) Cladotanytarsus pallidus Kieffer Cladotanytarsus vanderwulpi (Edwards) Lithotanytarsus dadesi Reiss Micropsectra atrofasciata (Kieffer) Micropsectra bidentata (Goetghebuer) Micropsectra contracta Reiss Micropsectra junci (Mg.)

Micropsectra lindrothi Goetghebuer Paratanytarsus bituberculatus (Edwards) Paratanytarsus confusus Paimén Paratanytarsus dissimilis Johannsen Paratanytarsus inopertus (Walker) $P$. mediterraneus Reiss \& Säwedal Paratanytarsus tenellulus (Goetghebuer) Paratanytarsus tenuis (Mg.)

Rheotanytarsus ceratophylli Dejoux Rheotanytarsus curtistylus (Goetghebuer) Rheotanytarsus muscicola Thienemann Rheotanytarsus pellucidus (walker) Rheotanytarsus pentapoda (Kieffer) Rheotanytarsus photophilus (Goetghebuer) Rheotanytarsus procerus Reiss Rheotanytarsus reissi Lehmann Rheotanytarsus ringei Lehmann Stempellina almi Brundin Tanytarsus brundini Lindeberg Tanytarsus chinyensis Goetghebuer Tanytarsus cretensis Reiss Tanytarsus ? fimbriatus Reiss \& Fittkau Tanytarsus gregarius Kieffer Tanytarsus heusdensis Goetghebuer Tanytarsus homi Goetghebuer Tanytarsus medius Reiss \& Fittkau Tanytarsus palettaris Verneaux Tanytarsus separabilis Brundin Tanytarsus signatus (v.d.W.)

Tanytarsus sp Virgatanytarsus albisutus (Cranston\&Armitage) Virgatanytarsus ansatus Reiss \& Schürch Virgatanytarsus arduennensis (Goetghebuer) Virgatanytarsus triangularis (Goetghebuer) Virgatanytarsus Pe 1 Langton 1991
Tableau 1. Suite.

Table 1. Continued.

$$
\begin{gathered}
1,2,3 \\
2 \\
2
\end{gathered}
$$

1,2

2,7 10,13

10 1

1, 2 
que le Haut-Atlas renferme un maximum d'espèces en Orthocladiinae.

Les Orthocladiinae et les Tanytarsini sont généralement rhéophiles, d'où leur fréquence au nord où les rivières ont des pentes plus ou moins aiguës. Les Chironomini sont plutôt fréquents dans les eaux plus lentiques et soumises à des pollutions, ce qui explique leur dominance d'une part, dans l'oued Sebou, l'oued Fès et l'oued Bouferkane du Moyen-Atlas qui présentent des eaux polluées en certaines zones et d'autre part, à cause des prospections plus fréquentes dans les rivières de plaine. La dominance des Diamesinae dans cette aire ainsi que dans le Haut-Atlas ne résulterait que des prospections qui furent dans des sites de haute altitude tels que des lacs et certains sites du réseau hydrographique de l'oued Oum-er-Rbia du Moyen-Atlas et dans celui de l'oued Tensift, les Gorges de Todra et la source de M'Goum du Haut-Atlas. L'Anti-Atlas renferme très peu d'espèces, ce qui est dû vraisemblablement à la rareté des prospections dans cette aire.

Les tendances biogéographiques montrent que $62 \%$ des espèces récoltées dans le Rif sont paléarctiques, $19 \%$ holarctiques et $7 \%$ méditerranéennes, ce qui souligne une influence nette de l'aire paléarctique sur le domaine rifain. Tandis qu'une tendance vers l'aire afrotropicale se manifeste pour les régions plus méridionales du Maroc.

Aux dix espèces marocaines à répartition circumméditerranéennes citées par Azzouzi et al. (1992), on peut ajouter trois espèces :

Paramerina spec. Griechenland : N-Maroc, Grèce, Espagne, Italie.

Cricotopus levantinus : N-Maroc, Liban.

Parakiefferiella wuelkeri : N-Maroc, S-Maroc, Tunisie, Espagne.

De même, aux autres espèces marocaines signalées comme endémiques de la région Sud-Ouest paléarctique par Azzouzi et al. (1992), on peut ajouter une espèce et six nouveaux taxa :

Procladius Pe3 Langton 1991 : N-Maroc, Sud Péninsule Ibérique (Calle et al. 1995).

Limnophyes ninae : N-Maroc.

Parametriocnemus Pe1 Langton 1991 : N-Maroc.

Cryptochironomus Pe5 Langton 1991 : N-Maroc.

Demicryptochironomus Pel Langton 1991 : N-Maroc, Tunisie (Boumaiza \& Laville 1988).

Stictochironomus Pe2 Langton 1991 : N- Maroc, Tunisie (Boumaiza \& Laville 1988), Sud Péninsule Ibérique (Calle et al. 1995).

\section{Virgatanytarsus Pe1 Langton 1991 : N-Maroc.}

En conclusion, les différentes aires prospectées témoignent d'une importante richesse spécifique en Diptères Chironomidés avec actuellement 317 espèces ou taxa. La localisation du Maroc entre l'Europe et l'Afrique favorise d'héberger un peuplement chironomidien mixte avec des influences paléarctiques et afrotropicales. Cette richesse n'est que le résultat de la grande diversité des milieux offerts par la géologie du Maroc. Toutefois, la potentialité d'une faune chironomidienne encore plus riche dans les réseaux hydrographiques de l'ensemble du pays encore peu étudiés doit être abordée d'une manière plus intensive sur tout le territoire marocain.

\section{Remerciements}

Les auteurs remercient les Drs Peter Langton (Coleraine, NIreland) et Henri Laville (Toulouse, France) pour la lecture et les corrections du premier manuscrit.

\section{Travaux cités}

Azzouzi A. \& Laville H. 1987. - Premier inventaire faunistique des Chironomidés (Diptera, Chironomidae) du Maroc. Ann. Limnol., 23 (3) : 217-224.

Azzouzi A., Laville H. \& Reiss F. 1992. - Nouvelles récoltes de Chironomidés (Diptera) du Maroc. Ann. Limnol., 28 (3) : 225-232.

Boumaiza M. \& Laville H. 1988. - Premier inventaire faunistique (Diptera, Chironomidae) des eaux courantes de la Tunisie. Ann. Limnol., 24 (2) : 173-181.

Calle D., Vilchez A. \& Casas J. 1995. - Les Chironomidés (Diptera) du bassin du Haut-Guadalquivir (Sierra de Cazorla, sud de l'Espagne). Ann. Limnol., 31 (3) : 201-213.

El Mezdi Z. \& Giudicelli J. 1985. — Etude d'un écosystème limnique peu connu : Les Khettaras de la région de Marrakech (Maroc), Habitats et Peuplements. Sciences de l'eau, 6 (3) : 281-297.

Kettani K., Vilchez A., Calle D. \& El Ouazzani T. 1994 _ Les Chironomidés (Diptera) de l'Oued Laou (Versant méditerranéen du Rif, Maroc). Ann. Limnol., 30 (1) : 25-32.

Kettani K., Vilchez A., Calle D. \& El Ouazzani T. 1995. - Nouvelles récoltes de Chironomidés (Diptera) du Maroc : Les Chironomidae de l'Oued Martil (Rif). Ann. Limnol., 31 (1) : 253-261.

Kettani K., El Ouazzani T. \& Calle D. 1997. - Nuevas especies de Quironomidos (Insecta : Diptera) en el Alto Laou (Rif, norte de Marruecos). Zoologica Baetica, $8: 181-189$.

Langton P.H. 1991. - A key to pupal exuviae of West Palaearctic Chironomidae. Privately published, 5 Kylebeg Av. Coleraine, Co Londonderry, N.-Ireland : 386p.

Laville H. 1981. - Récoltes d'exuvies de Chironomidés (Diptera) dans le Haut-Lot de la source $(1295 \mathrm{~m})$ au confluent de la Truyère (223 m). Ann. Limnol., 17 (3) : 225-289.

Naya A. 1988. - Peuplements chironomidiens (Diptera) du bassin du Haut et Moyen Sebou : Biotypologie et valeurs Bio-indicatrices. Thèse $3^{\text {eme }}$ cycle. Univ. Mohammed V. Rabat (Maroc) : $127 \mathrm{p}$.

Pique A. - Géologie du Maroc : 278p.

Rhamdani M. \& Tourenq J.N. 1982. - Contribution à l'étude faunistique de la Merja de Sidi Bou Ghaba. Bull. Ins. Sci., Rabat, $\mathrm{n}^{\circ}$ $6: 179-223$.

Thauvin J.P. 1971. - Présentation du domaine rifain. Notes et Mémoires du service géologique. No. $231: 27-80$. 